\title{
On Mechanics of Deformation and Crushing Processes
}

\author{
L. Berka ${ }^{1, a}$ \\ ${ }^{1}$ Czech Technical University, Faculty of Civil Engineering, Department of Building Structures, \\ Prague, Czech Republic \\ ${ }^{a}$ berka@fsv.cvut.cz
}

The mechanics of crushing and breaking of particles is one of the most intractable problems in materials science. The stressed states of processed materials are significantly inhomogeneous, and thus the deformation and disintegration mechanisms vary greatly. Two techniques have been developed for realizing these processes as a quasi-homogeneous transition. The device and method developed by Enikolopov transform a solid polymer spontaneously into powder. The same loading system is now used for obtaining fine-grained metals, similarly as when using the ECAP device developed by Valiev. Both techniques are now used for obtaining nanostructured materials. The common feature of both types of methods is the formation of new physical surfaces. These are particle-free oversurfaces or grain boundaries. The method requires a supply of energy in the form of mechanical work, and this is mostly done by simultaneous action of pressure and shear stress. The formation of free oversurfaces in stressed solid bodies is the subject of fracture mechanics. The Griffith equation is employed to describe the problem.

Keywords: technology, processes, solids, crushing, mechanics, polar continuum, particle, oversurface, grain, grain boundary.

Introduction. Granulation forms the basis of many mechanical technologies that aim to change the material substructure into a bulk. Two techniques have been developed for realizing these processes as a quasi-homogeneous transition [1, 2]. The device and method developed by Enikolopov [3] transforms a solid polymer spontaneously into powder. The same loading system is now used for obtaining fine-grained polycrystals, similarly as when using the ECAP device [4] developed by Valiev. Both techniques are now used for obtaining nanostructured materials [5]. It is necessary to clarify the micromechanisms of these processes for the purposes of materials science as well as practical applications.

The initial phase of the processes in question is the state with large deformations where local rotation as a part of the deformation gradient cannot be neglected. This effect has been studied experimentally using $\mathrm{X}$-ray techniques $[6,7]$ and also microscopically on the polished surface of specimens [8-10]. The theoretical analysis of deformations assuming local rotations is known as the Cosserat continuum theory. This theory was developed in the second half of the 20th century $[11,12]$. A number of problems involving formation of the substructure and grain size were solved using a coupled stress theory $[13,14]$.

The second phase of the spontaneous fragmentation process of quasi-homogeneous solids, which takes place under pressure and shear stress, results in the formation of new physical surfaces, i.e., particle-free oversurfaces [3] and a new grain structure with new grain boundaries [4]. The limit states of individual shear cracks and shear bands are now under very intensive theoretical and experimental study. An early paper [15] provided an elastic solution for the in-plane crack problem as well as the out-of-plane crack problem. 3D shear cracks were studied in [16-18] using the extended finite element method and continuum-discontinuum modeling.

A description of the final state of the process now arises from the superposition of the two phases. This shows the formation of the field of deformations and rotations and shear spherical cracks that they induce. The deformation field around a spherical (C) L. BERKA, 2008 
macrocrack was studied in [19], using an interaction energy integral method. Attempts to solve the above mentioned problems are based on the assumption of a homogeneous continuum with individual defects $[20,21]$. The aim of this paper is to put forward a model of the granulation process, in which a quasi-homogeneous solid changes its grain size and structure in the whole body volume.

A Model of the Granulation Process in a Solid Body. The general principle of an idealized granulation process is a transition of a homogeneous solid body into a bulk of homogeneous particles having surface tension and the same mass as in the original body. The process is comparable with brittle fracture, but the stress states and crack modes are different. Brittle fracture takes place mainly under tensile stress and in the form of the first crack opening mode. According to the experimental results introduced above, the analyzed process continues under the combination of both shear and pressure stresses. This stress state then results in cracks of the 2 nd and 3 rd modes. The energy balance principle used in the study of crack problems is expressed by the Griffith equation:

$$
d E=d S+d U-d W=0, \quad d W=2 d U
$$

where $E$ is the total potential energy of a cracked body, $S$ is the surface energy of the crack, $U$ is the strain energy of a deformed body with a crack, and $W$ is the potential energy of the applied loads. The presented relation between $W$ and $U$ is valid on condition that the strains are elastic.

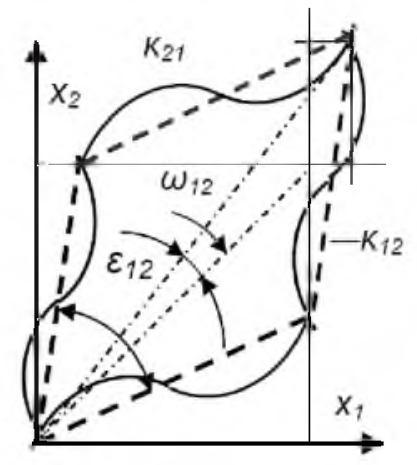

Fig. 1. system of deformations [11].

The Polar Continuum Mechanics Equations. Deformations in technological processes are always large and the experimental results introduced above show that in theoretical description kinematic rotations of a deformed material cannot be neglected. The effect of local rotation is described in the mechanics of deformable bodies by micropolar continuum theories. There, the whole system of deformations (Fig. 1) and stresses is supplemented by the introduction of the moment stress tensor $\mu_{i j}$ and distortion tensor $\kappa_{i j}$ into the equations that describe its mechanical behavior. The deformation of the differential representative volume element of a material is expressed by the following relations [11]:

$$
\begin{aligned}
& d u_{i}=u_{i, j} d x_{j}=\varepsilon_{i j} d x_{j}+\omega_{i j} x_{j}, \\
& \varepsilon_{i j}=1 / 2\left(u_{i, j}+u_{j, i}\right), \quad \omega_{i j}=1 / 2\left(u_{i, j}-u_{j, i}\right), \\
& \omega_{i}=\varepsilon_{i j k} \omega_{i j}, \quad d \omega_{i}=\omega_{i, j} d x_{j}=\kappa_{i j} d x_{j} .
\end{aligned}
$$

The stresses $\sigma_{i j}$ and $m_{i j}$ that occur in the centers of the volume element planes are described by the following equations: 


$$
\sigma_{i j, j}=0, \quad \sigma_{n m}-\sigma_{n m}+\varepsilon_{i m n} \mu_{i j, j}=0 .
$$

The elastic behavior of isotropic materials is then defined by the following relations:

$$
\begin{aligned}
& s_{i j}=2 G\left(\frac{v}{1-2 v} \varepsilon \delta_{i j}+\varepsilon_{i j}\right), \quad s_{i j}=\frac{1}{2}\left(\sigma_{i j}+\sigma_{j i}\right), \quad \varepsilon=\varepsilon_{i i}, \\
& m_{i j}=4 a^{2} G\left(\kappa_{i j}+\eta \kappa_{j i}\right), \quad \kappa_{i j}=\frac{m_{i j}-\eta m_{j i}}{4 a^{2} G},
\end{aligned}
$$

where $G$ and $v$ are elastic constants and $a$ and $\eta$ are parameters of the material substructure.

The strain energy $d U$ in the differential volume element $d V$ is determined by the following formula:

$$
d U=u d V, \quad u=s_{i j} \varepsilon_{i j}+m_{i j} \kappa_{i j} .
$$

Spherical Shear Cracks in a Spatially Compressed Material. A crucial point in the granulation process is connected with the origin of the spherical particle form. It is necessary to take into account the continuous field of local rotations resulting from large shear strain. Experimental results show that at a certain level of the shear strain, rotations stay discontinuous [6]. When the material is assumed to be isotropic, the originating discontinuities acquire the form of a sphere as these rotations are always spatial. Furthermore, this transition is possible owing to the energy flux coming from the compressed volume elements into the surface layers and originating along the spherical discontinuities, which can be seen as frozen eddies with boundary layers. To reach the conditions for the transition limit state, the Griffith equation is used. The quantities of the surface energy $S$ and strain potential energy $U$, therefore, have to be determined.

Let us now assume spherical shear cracks on the surface of a sphere placed inside a representative volume element cube (Fig. 2). The cracks are bounded by circles, which originate from sections parallel to the sides of the cube. Their surface area is the same as that of the six spherical segments. The differentials of their surface area and volume are determined by the following formulas:

$$
d A=12 \pi R^{2} \sin \varphi d \varphi, \quad d V=6 \pi R^{3} \sin ^{3} \varphi d \varphi,
$$

where $R$ is the radius of the sphere and $\varphi$ is the angle between its normal and meridian plane.

The crack surface energy $d S$ is now obtained by multiplying the crack surface area $2 d A$ by the specific surface energy $\gamma$, which also contains the energy of the plastically deformed surface layer.
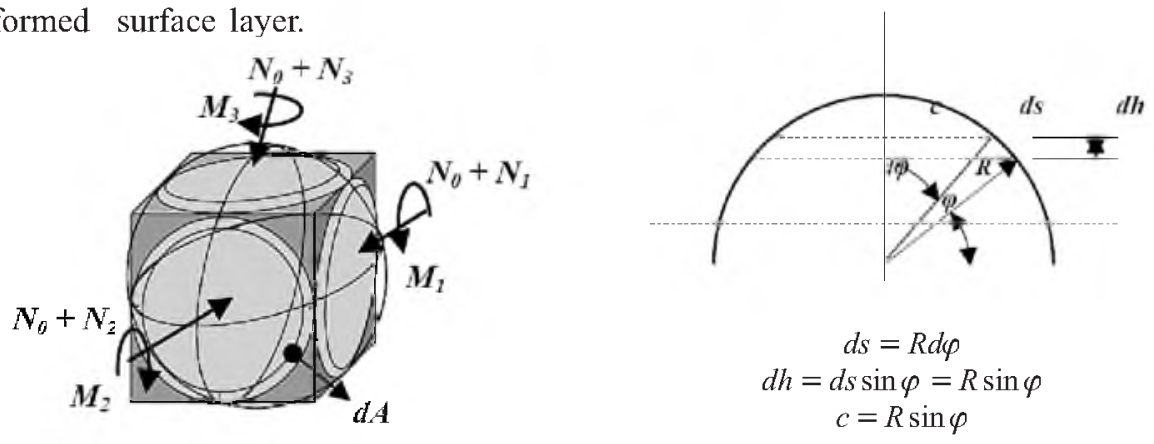

Fig. 2. Volume element cube. 
The strain energy density $u$ in the material with polar stresses is now calculated by the substitution of inverse relation (4) for $\kappa_{i j}$ in Eq. (5), and further arranged and expressed in the form containing the invariants $S_{()}$and $M_{O}$ of the stress and moment tensors $s_{i j}$ and $m_{i j}$ :

$$
\begin{aligned}
u & =\frac{s_{i j} s_{i j}-\frac{\mu}{3(1+\mu)} s_{i i} s_{j j}}{2 G}+\frac{m_{i j} m_{i j}-\eta m_{i j} m_{j i}}{4 a^{2} G\left(1-\eta^{2}\right)} \\
& =\frac{1}{2 G}\left[\frac{S_{(1)}^{2}}{3(1+\mu)}+S_{(2)}^{D}\right]+\frac{M_{(2)}^{A}-\eta M_{(2)}^{S}}{2 a^{2} G\left(1-\eta^{2}\right)} .
\end{aligned}
$$

The strain energy $d U$ is now expressed using Eqs. (5)-(7)

$$
d U=\left\{\frac{1}{2 G}\left[\frac{S_{(1)}^{2}}{3(1+\mu)}+S_{(2)}^{D}\right]+\frac{M_{(2)}^{A}-\eta M_{(2)}^{S}}{2 a^{2} G\left(1-\eta^{2}\right)}\right\} R^{3} \sin ^{3} \varphi d \varphi .
$$

Substituting both quantities $d S$ and $d U$ in the Griffith equation, Eq. (1) results in the following formula:

$$
d E=\left\{2 \gamma-\left\langle\frac{1}{2 G}\left[\frac{S_{(1)}^{2}}{3(1+\mu)}+S_{(2)}^{D}\right]+\frac{M_{(2)}^{4}-\eta M_{(2)}^{S}}{2 a^{2} G\left(1-\eta^{2}\right)}\right\rangle R \sin ^{2} \varphi\right\} 6 \pi R^{2} \sin \varphi=0 .
$$

An approximation is accepted for the purpose of simplifying the equation. The angle $\varphi$ is assumed for the octahedral plane, i.e., the value of $\sin ^{2} \varphi$ then equals $3 / 4$. The character of the parameters $a$ and $R$ should be pointed out. Both express the length and stand for the characteristic dimension of structural elements, i.e., the particle size. Therefore, an equation taking into account the condition $R=a$ leads, after some rearrangement, to the following quadratic form regarding the structural element size $a$ :

$$
a^{2}\left(1-\eta^{2}\right)\left[\frac{S_{(1)}^{2}}{1+\mu}+3 S_{(2)}^{D}\right]-16 \gamma a G\left(1-\eta^{2}\right)+3\left(M_{(2)}^{4}-\eta M_{(2)}^{S}\right)=0 .
$$

The solution of this form will be the subject of the next theoretical and experimental analyses.

Conclusions. The solution of the problem under study is based on the account of the mutual coupling of shear deformations with local rotations. The rotations predetermine the origin of shear spherical cracks in all internal points of the macrovolume of the material. The energy supplied to the system by the applied spherical pressure then leads, together with other physicochemical auxiliary effects, to new conditions for thermo- dynamic equilibrium of the process and to the formation of new physical surfaces.

Acknowledgment. The author appreciates the support of GA ASCR for the project No. IAA200710604.

1. V. V. Boldyrev and K. Tkáčová, J. Mater. Synth. Proc., 8, 121-132 (2000).

2. J. R. Kolobov and R. Z. Valiev, in: NAUKA, Novosibirsk (2001), p. 228. 
3. N. S. Enikolopian, Macromolec. Chem., 185, 1371-1381 (1984).

4. R. Z. Valiev, A. V. Korznikov, and R. R. Milyukov, Mater. Sci. Eng., A186, 141 (1993).

5. G. Wilde et al., Mater. Sci. Eng., A449-A451, 825-828 (2007).

6. B. M. Rovinskij and V. M. Sinajskij, Some Problems of Strength of Solids, Moscow (1958).

7. W. Diepolder,V. Mannl, and H. Lippman, Int. J. Plasticity, 7, No. 4, 313-328 (1991).

8. L. Berka, J. Mater. Sci., 19, No. 5, 1486-1495 (1982).

9. L. Berka, Proc. ICM 8, Univ. of Victoria B. C. (1992), Vol. I, pp. 160-164.

10. L. Berka, et al., Solid Mech. Appl., 135, 235-246 (2006).

11. R. D. Mindlin and H. F. Tiersten, Arch. Rat. Mech. Anal., 11, 415 (1962)

12. A. C. Eringen, in: H. Liebowitz (Ed.), Fracture, Academic Press (1968), Vol. II, pp. 621-729.

13. R. S. Lakes, in: H. Mühlhaus and J. Wiley (Eds.), Continuum Model for Materials with Microstructure (1995), pp. 1-22.

14. H. C. Parks and R. S. Lakes, Int. J. Solids Struct, 23, No. 4, 485-503 (1987).

15. G. I. Barenblatt and G. P. Cherepanov, J. Appl. Math. Mech., 25, 1654-1666 (1961).

16. G. C. Sih and H. Liebowitz, in: H. Liebowitz (Ed.), Fracture, Academic Press (1968), Vol. II, pp. 151-166.

17. N. Moes, A. Gravouil, and T. Belytschko, Int. J. Num. Meth., 2549-2568 (2002)

18. E. Samaniego and T. Belytschko, Int. J. Num. Meth., 62, 1857-1872 (2002)

19. M. Gosz and B. Moran, Eng. Fract. Mech., 69, 299-319 (2002).

20. S. Casolo, Int. J. Solids Struct., 43, 475-496 (2006).

21. E. Z. Wang and N. G. Shrive, Eng. Fract. Mech., 52, No. 6, 1107-1126 (1995).

Received 28.06.2007 